\title{
Uterine Lipoleiomyoma in a Young Female- A Rare Case Report with Review of Literature
}

\author{
Maneesh Goyal ${ }^{1}$, Anshu Gupta ${ }^{2}$, Sachin Bansal ${ }^{3}$, Rajni Bharti ${ }^{4}$, Anurag Gupta ${ }^{5}$. \\ ${ }^{1}$ Senior resident in Pathology, Emergency Laboratory, Institute of Human Behavior and Allied Sciences \\ (IHBAS), Delhi, India \\ ${ }^{2}$ Associate Professor in Pathology, Department of Pathology, Institute of Human Behavior and Allied Sciences \\ (IHBAS), Delhi, India \\ ${ }^{3}$ Senior resident in Pathology,Department of Pathology, Institute of Human Behavior and Allied \\ Sciences(IHBAS),Delhi, India \\ Professor in Pathology, Department of Pathology, Sarojini Naidu Medical College, Bhim Rao Ambedkar \\ University, Agra, India \\ Assistant Professor in Pathology, Department of Pathology, Sarojini Naidu Medical College, Bhim Rao \\ Ambedkar University, Agra, India
}

\begin{abstract}
Lipoleiomyoma is a rare benign uterine tumour consisting of smooth muscles and mature adipose tissue. Most cases of lipoleiomyomas cannot be distinguished clinically from leiomyoma and to a large extent diagnosis can be made on the typical gross appearance of the tumour. Incidence of this neoplasm is estimated to be 0.03-0.2\%. The majority of patients do not report any symptoms. Almost all of the cases of lipoleiomyoma have been reported in postmenopausal women and predominantly located in the uterus; however, extrauterine locations have been reported as well. We are reporting a case of lipoleiomyoma of uterus in a 28 yrs old female. To the best of our knowledge, this is the only case reported of lipoleiomyoma of uterus at such an early age.
\end{abstract}

Keywords: lipoleiomyoma, leiomyoma, uterine tumor.

\section{Introduction}

Uterine lipoleiomyoma is a rare benign uterine tumour consisting of smooth muscles and mature adipose tissue. The reported incidence varies from $0.03 \%$ to $0.2 \%{ }^{1}$. Contrary to uterine leiomyomas which are usually found in women at the reproductive age and regress after menopause, lipoleiomyoma is more frequently observed in older patients. Most of the patients are postmenopausal predominantly in their fifties and sixties ${ }^{2}$. Uterine lipoleiomyomas are associated with ordinary leiomyomas. The sign and symtoms are also similar to those caused by ordinary leiomyomas of the same size. Most patients are asymptomatic ${ }^{3}$. Uterine lipoleiomyomas are most frequently found in the body of uterus and are usually intramural. Lipoleiomyomas can be found anywhere in the uterus or cervix and may be subserosal ${ }^{4}$. On USG, leiomyomas appears as hyperechoic mass partially encased by a hypoechoic rim. The rim is thought to represent a layer of myometrium surrounding the fatty component ${ }^{1}$. The fatty component can be confirmed on MRI using fat-suppression techniques.Fatty metamorphosis of smooth muscle cells of leiomyomas is the most likely cause for the development of lipoleiomyomas ${ }^{5}$.

\section{Case Study}

The patient presented to us was a 28 yrs old female with chief complaints of menorrhagia and pelvic pain of few months duration. Clinical examination revealed bulky uterus with firm consistency. USG examination of the pelvis showed an enlarged uterus with a well defined echogenic mass in the uterine wall distorting the uterine cavity. It measured about $3.1 \times 2.2 \mathrm{~cm}$ in size with some acoustic shadows. Further examination with CT scan showed a minimally enhancing fat density lesion which measured about $3.24 \times 2.28 \times 1.66 \mathrm{~cm}$ in size located at the lateral uterine wall. Patient was taken up for exploratory laparotomy which revealed bulky uterus. Both tubes and ovaries appeared to be normal. Total hysterectomy was done while conserving both ovaries of the patient.

Grossly, on cut section the specimen of the uterus showed an intramural as well as endophytic mass measuring $3.1 \times 1.6 \mathrm{~cm}$ involving body and lateral uterine wall, distorting the uterine cavity (Fig. 1). The mass was soft to firm in consistency and cut surface was yellowish gray and intersected by bands of grayish white fibrous tissue. Areas of hemorrhage were also seen. Serosal surface of the uterus was unremarkable. Microscopically, Hematoxylin and Eosin (H\& E) stained sections of the uterine mass showed mainly lobules of mature adipose tissue with intervening smooth muscle cells and fibrous septae (Fig. 2, 3). Bizarre pleomorphic cells, mitotic figures or necrosis was not present. 


\section{Discussion}

Uterine lipoleiomyomas are rare in gynecological practices. They should be considered, however in differentiating palpable lesions in the uterus, especially in postmenopausal women ${ }^{2,6}$. Case with complex diagnostics, with the use of USG, as well as CT and MRI, when lipoleiomyoma is suspected, are extremely rare. Uterine lipoleiomyoma is a rare benign neoplasm consisting of an intimate admixture of mature adipocytes, smooth muscles and fibrous tissue. The reported incidence varies from $0.03 \%$ to $0.2 \%{ }^{1}$. Utrine lipoleiomyoma is typically seen in postmenopausal women predominantly in their fifties and sixties. They are associated with ordinary leiomyomas. In one study, 10 out of 17 cases of lipoleiomyomas were associated with ordinary leiomyoma ${ }^{7}$. The sign and symtoms are also similar to those caused by ordinary leiomyomas of the same size. Most patients are asymptomatic. In postmenopausal women, suffering from hypothyroidism, diabetes or hyperlipidemia, more frequently lipoleiomyoma like changes in the uterus are found ${ }^{8}$. It is suggested that metabolic changes occurring in postmenopausal women are a likely cause for the development of adipose tissue metamorphosis in lipoleiomyoma. In this case patient did not exhibit any metabolic disorder. Uterine lipoleiomyomas are most frequently found in the body of uterus and are usually intramural, in our case tumor present in corpus and lateral wall of uterus. The mass may be endophytic or exophytic, with respect to the uterus, endophytic in our case. On USG, leiomyomas appears as hyperechoic mass partially encased by a hypoechoic rim. The rim is thought to represent a layer of myometrium surrounding the fatty component ${ }^{1}$. The fatty component can be confirmed on MRI using fat-suppression techniques ${ }^{5}$. On gross, tumours are rounded or oval, well encapsulated, yellow and soft. Microscopically tumour shows lobules of mature adipose tissue and smooth muscles intersected by fibrous septae. There is much speculation on the histogenesis of uterine lipoleiomyomas because adipose tissue does not occur in the myometrium. The various theories advanced include misplaced embryonic fat cells, metaplasia of muscle or connective tissue, perivascular extension of fat along uterine vessels and origin from pleuripotent cells and embryonic multipotential component. Some others advocate the possibility that these growths are mixed tumours that arise from immature cells with multiple potentialities for differentiation. Salm suggested that these tumours should be considered as fatty metaplasia in leiomyoma. Another theory was that fat cells proliferate into the uterus from the subserosal fat of neighboring structures and also from perivascular fat cells accompanying the blood vessels into the uterus. In most of the cases the diagnosis is made on the gross appearance as these tumours have the typical yellow greasy appearance of adipose tissue with intermingled smooth muscle fibers and fibrous septae. Differential diagnosis of the lipomatous mass in the pelvis includes: benign cystic ovarian teratoma, malignant degeneration of cystic teratoma, nonteratomatous lipomatous ovarian tumor, benign pelvic lipoma. Imaging plays an important role in preoperative identification of the fatty nature and exact intrauterine location of a leiomyoma. Liposarcoma and lipoblastic lymphadenopathy, associations of lipomatous uterine tumors and endometrial carcinoma with lipoleiomyosarcoma arising in uterine lipoleiomyoma have been reported ${ }^{8}$.

\section{Figures}

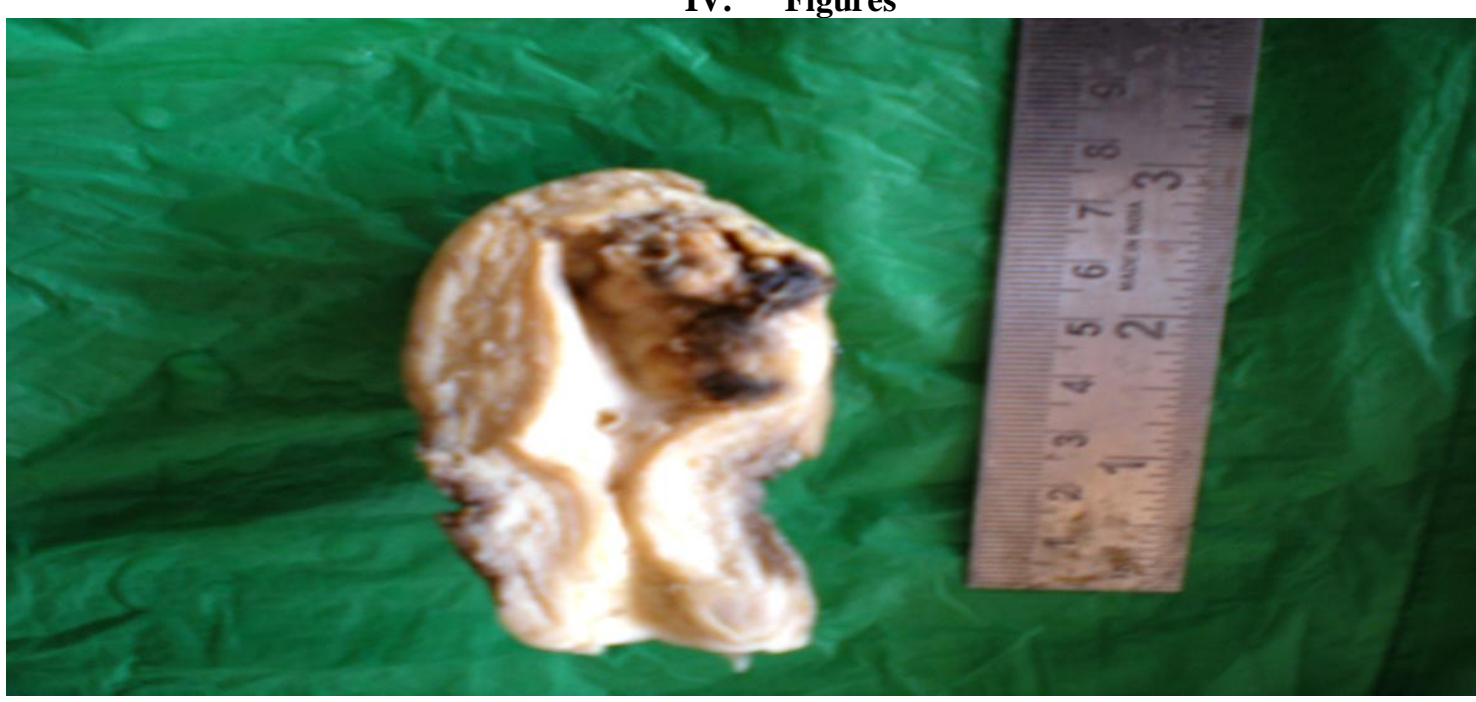

Figure 1. Gross specimen of the uterus showing an intramural and endophytic mass distorting the uterine cavity with hemorrhagic areas on external aspect. 


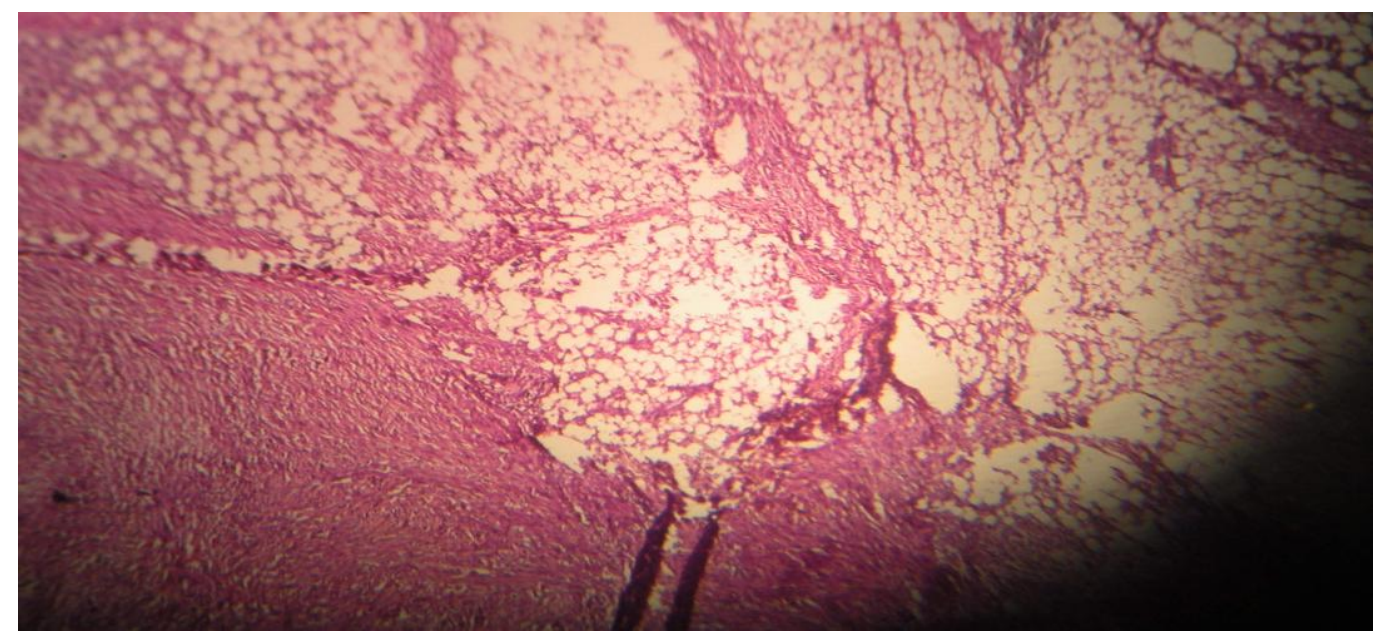

Figure 2. Photomicrograph of the uterine mass showing lobules of mature adipose tissue intermingled with the fascicles of smooth muscle cells (H\& E x 10x).

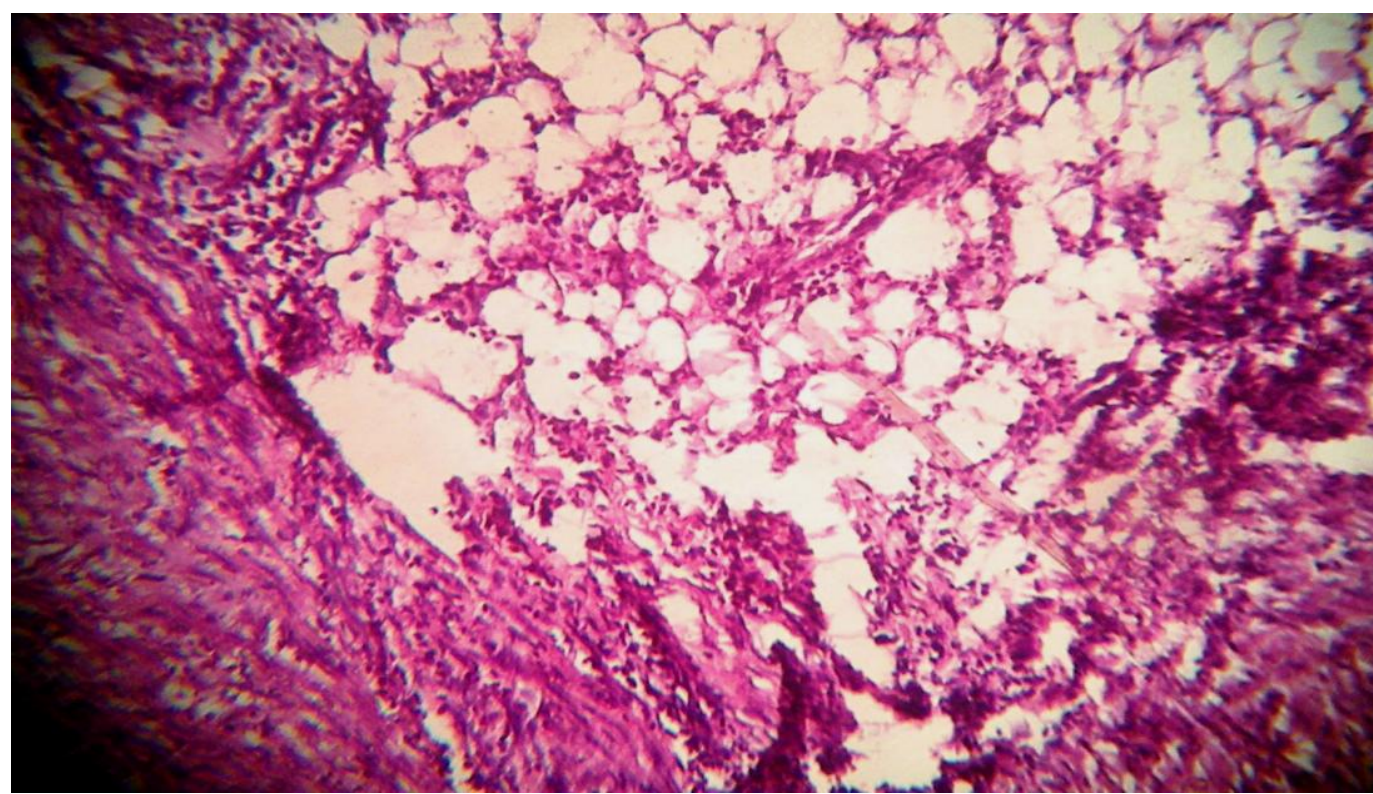

Figure 3. Photomicrograph of the uterine mass showing lobules of mature adipose tissue intermingled with the fascicles of smooth muscle cells ( $\mathrm{H} \& \mathrm{E} \mathrm{x}$ 40x).

\section{Conclusion}

When palpable mass is detected during a manual pelvic examination in postmenopausal patients, a gynecologist should consider the possibility of lipoleiomyoma. USG images, CT and MRI may be helpful in preoperative diagnosis of these lesions. The final diagnosis is established on the basis of a histopathological examination of the tissue specimen.

\section{References}

[1]. A. Prieto, C. Crespo, A. Pardo, I. Docal, J. Calzada, P. Alonso, Uterine lipoleiomyomas: US and CT findings, Abdom Imaging, 25, $2000,655-657$.

[2]. L.M. Kauser, C.H. Carrasco, C.R. Sheehan, et al, Lipomatous tumour of the uterus; Radiographic and ultrasonic appearance, $\mathrm{Br} \mathrm{J}$ Radiol, 52, 1979, 992-993.

[3]. D. A. Oppenheimer, B. A. Carroll, S.W. Young, Lipoleiomyoma of uterus, J Comput Assist Tomogr, 6, 1982, 640-642.

[4]. R. Aizenstein, A. C. Wilbur, S. Aizenstein, CT and MRI of uterine lipoleiomyoma, Gynecol Oncol, 40, 1991, 274-276.

[5]. Y. Tsushima, T. Kita, K. Yamamoto, Uterine lipoleiomyomas: MRI, CT and Ultrasonographic findings, Br J Radiol, 70, 1997, 10681070.

[6]. S. Sudhamani, Durgaprasad Agrawal, Ajita Pandit, V. M. Kiri, Lipoleiomyoma of uterus: a case report with review of literature, Indian J. Pathol. Microbiol., 53(4), 2010, 840-841.

[7]. Rony Avritscher, Revathy B. Iyer, Jae Ro, Gary Whitman, Lipoleiomyoma of the Uterus, Radiologic-Pathologic conferences of the University of Texas, M. D. Anderson Cancer Center, AJR, 177, 2001, 856.

[8]. K. C. Lin, B. C. Sheu, S. C. Huang, Lipoleiomyoma of the uterus, Int. J. Gynaecol. Obstet., 67(1), 1999, 47-49. 